\title{
Density functional theory study explaining the underperformance of copper oxides as photovoltaic absorbers
}

\author{
Aleksandar Živković, Alberto Roldan, and Nora H. de Leeuw* \\ Cardiff University, School of Chemistry, Main Building Park Place, Cardiff CF10 3AT, United Kingdom
}

(Received 9 August 2018; revised manuscript received 24 December 2018; published 28 January 2019)

\begin{abstract}
Most photovoltaic absorbers are identified using the standard Shockley-Queisser selection principle which relies on optimal band-gap values. However, this criterion has been shown to be insufficient, as many materials with appropriate values still perform badly. Here, we have employed calculations based on the density functional theory to assess three copper oxides as potential photovoltaic materials: $\mathrm{Cu}_{2} \mathrm{O}, \mathrm{Cu}_{4} \mathrm{O}_{3}$, and $\mathrm{CuO}$. Despite their promising theoretical solar power conversion efficiency of over $20 \%$, experimental values are found to be far lower. Theoretical evaluation of the electronic and optical properties reveals that certain transitions within the band structures are dipole forbidden, whereas the fundamental band gaps of $\mathrm{Cu}_{4} \mathrm{O}_{3}$ and $\mathrm{CuO}$ are of indirect nature. These findings correlate to the weak and shifted absorption properties found experimentally, which underpin the inefficient light capture by copper oxides. Based on these results and an applied extended selection metric, we can explain why copper oxides are unable to reach the efficiencies previously proposed theoretically and why we need to revise their maximum conversion values.
\end{abstract}

DOI: 10.1103/PhysRevB.99.035154

\section{INTRODUCTION}

Copper is found as three different binary oxides: $\mathrm{Cu}_{2} \mathrm{O}$ (cuprous oxide, also known as the mineral cuprite), $\mathrm{CuO}$ (cupric oxide or tenorite), and $\mathrm{Cu}_{4} \mathrm{O}_{3}$ (paramelaconite). Throughout history, copper oxides have been studied thoroughly, including both theoretical and experimental works, especially because their unique electronic and optical properties allow their potential applicability as transparent conducting oxides (TCOs) [1]. They are $p$-type semiconducting materials with band-gap values that make them suitable for photovoltaic (PV) applications [2]. In addition, copper oxides are abundant, nontoxic, and have low extraction costs [3].

$\mathrm{Cu}_{2} \mathrm{O}$ is a well examined binary oxide of copper, especially for harvesting solar energy. Schottky junctions, homojunctions, and heterojunctions up to nanocomposite structures based on $\mathrm{Cu}_{2} \mathrm{O}$ have all been studied extensively during the last two decades. However, the largest reported solar power conversion efficiency (PCE) has not exceeded 1\%, and solar cells based on $\mathrm{CuO}$ and especially $\mathrm{Cu}_{4} \mathrm{O}_{3}$ have not achieved noticeable photovoltaic performances yet either. Solar cells based on $\mathrm{CuO}$ thin-film heterojunctions with amorphous silica have reached only 3\% PCE [4], despite their almost ideal band-gap value which lies in the visible part of the electromagnetic spectrum [5]. So far, only $\mathrm{Cu}_{2} \mathrm{O}$ has reached reasonable efficiencies of 5-6\%, being part of multilayer solar cell devices [6].

The main reason that copper oxides are still attracting the attention of the scientific community is owing to the theoretical PCE of $\mathrm{Cu}_{2} \mathrm{O}$ and $\mathrm{CuO}$, which is estimated using the Shockley and Queisser (SQ) [7] predictor to be $20 \%$ and $30 \%$, respectively [8]. Although, to the best of our knowledge,

*Corresponding author: deleeuwn@ cardiff.ac.uk data for the PCE of $\mathrm{Cu}_{4} \mathrm{O}_{3}$ do not exist, some authors have suggested that it could be a better absorbing material than the previously characterized copper materials [9]. Following those promising arguments, the inevitable question arises why copper oxides still fail to display good photovoltaic absorbing efficiencies.

PV efficiencies result from a complex interplay of structural, thermodynamic, magnetic, electronic, and optical characteristics. Modeling of those values computationally presents a challenge due to the large number of theories, equations, and approximations that one needs to take into account to obtain the whole picture.

Traditional first-principle methods employed for the theoretical study of copper oxides range from basic density functional theory (DFT) within the local-density approximation (LDA) [10], via parametrized approaches (Hubbard's $+U$ approach [11]), to more sophisticated hybrid functionals (HSE06 [12], B3LYP [13], PBE0 [14]) and studies using Green's functions and the screened Coulomb interaction $[15,16]$ (so-called $G W: G_{0} W_{0}(\mathrm{LDA})$ [17], $G W^{R P A}$ [18], $s c G W^{R P A}$ [18], $G W^{L F}+V_{d}(\mathrm{GGA}+U)$ [19]). Basic DFT (using LDA and GGA) has failed to predict the electronic and magnetic structures of all three copper oxides, giving rise to metallic and non-magnetic $\mathrm{CuO}$ and $\mathrm{Cu}_{4} \mathrm{O}_{3}$, and a very low band gap for $\mathrm{Cu}_{2} \mathrm{O}(0.5 \mathrm{eV}$ [10]). The main modeling issue with those $\mathrm{CuO}$ and $\mathrm{Cu}_{4} \mathrm{O}_{3}$ calculations lies within the strong hybridization between copper $d$ and oxygen $p$ orbitals. Supplementing DFT by an on-site Coulomb correction $(\mathrm{DFT}+U)$ allows one to tune the band gap of $\mathrm{CuO}$ and $\mathrm{Cu}_{4} \mathrm{O}_{3}$, although the band gap of $\mathrm{Cu}_{2} \mathrm{O}$ remains severely underestimated. DFT $+U$ was created in order to describe the ordered ground state of correlated Mott/charge-transfer insulators such as $\mathrm{CuO}$ and $\mathrm{Cu}_{4} \mathrm{O}_{3}$ [19]. However, $\mathrm{Cu}_{2} \mathrm{O}$ is a band insulator, and does not fall into this category [20]. Hybrid functionals give excellent structural results, but yield 
overestimated band gaps of strongly correlated materials like $\mathrm{CuO}$ and $\mathrm{Cu}_{4} \mathrm{O}_{3}$ [12], whereas recent work of Rödl et al. [21] showed that electronic structure calculations of $\mathrm{CuO}$ are extremely sensitive to details of employed $G W$ iteration schemes. Wang et al. [19] performed GW calculations including DFT-derived local field effects $\left(G W^{L F}\right)$ with an additional on-site potential $\left(+V_{d}\right)$ to lower the $d$-orbital energies that are systematically located too high. This approach reproduced the band-gap values of $\mathrm{Cu}_{2} \mathrm{O}$ and $\mathrm{CuO}$ rather well, while leaving the value for $\mathrm{Cu}_{4} \mathrm{O}_{3}$ too low. Although the $G W$ method seems accurate enough, it is computationally rather expensive, and it still needs to be evaluated whether it can predict properties of unknown compounds, e.g., doped materials, nanostructures, or junctions.

As we are aware of the detailed work that has already been carried out on all three copper oxides, and in particular $\mathrm{CuO}$ and $\mathrm{Cu}_{2} \mathrm{O}$, the aim of this paper is not to duplicate existing data, but rather to define an affordable approach and address the missing parts that could help explain some of the major questions still open in this area. In the present work we have performed first-principle calculations within a set of unique approximations, but relying on the minimal number of additional parameters, for the description of all three oxides of copper. Furthermore, we have assessed their mechanical, electronic, magnetic, and optical properties and linked them to theoretical values of photovoltaic efficiencies. In addition, it was our aim to extend the standard SQ limit and obtain insight into the reason why copper oxides fail to achieve high solar-absorbing photovoltaic efficiencies, despite their promising energy band-gap values.

\section{COMPUTATIONAL METHODS}

We have performed density functional theory (DFT) calculations of all three copper oxides using the Vienna Ab Initio Simulation Package (VASP) code [24,25] with a plane-wave basis set and PAW potentials [26,27]. The Perdew-BurkeErnzerhof (PBE) general gradient approximation (GGA) [28] for the exchange-correlation $(\mathrm{XC})$ functional was used for both DFT and DFT $+U$ [29-32], while the hybrid-DFT XC functional was used with an additional $25 \%$ of the exact Hartree-Fock (HF) exchange (HSE06) [33-35]. The total energy was required to converge to within $10^{-5} \mathrm{eV}$, and the interatomic forces were minimized to less than $0.01 \mathrm{eV} / \AA$ for structural relaxations.

For the Brillouin-zone sampling, a $\Gamma$-centered MonkhorstPack $k$ mesh was used [36]. Long-distance dispersion corrections were included using the D3 approach of Grimme et al. [37] and spin polarization was taken into account. Convergence of the size of the plane-wave basis and the number of $k$ points employed was tested independently for $\mathrm{Cu}_{2} \mathrm{O}$, $\mathrm{CuO}$, and $\mathrm{Cu}_{4} \mathrm{O}_{3}$. A cutoff energy of $500 \mathrm{eV}$ together with $7 \times 7 \times 7,5 \times 5 \times 5$, and $5 \times 5 \times 3$ meshes, respectively, was sufficient to reach convergence. The total energy was required to converge to within $10^{-5} \mathrm{eV}$, and the interatomic forces were minimized to less than $0.01 \mathrm{eV} / \AA$ for structural relaxations. Band structure and density of states (DOS) calculations were performed along high-symmetry directions obtained from the Bilbao Crystallographic Server [38-40]. The band structures were plotted using the Wannier90 code
[41]. Crystal structure drawings were produced with VESTA [42]. Due to the increasing computational cost of our proposed approach, and in order to compare structural and mechanical properties, a simplified arrangement of $\mathrm{CuO}$ spins was adopted, rather than the fully extended model proposed by Forsyth et al. [43]. Values for the effective Hubbard parameter $U_{\text {eff }}$ were applied on the localized $3 d$ electrons of $\mathrm{Cu}$, and systematically varied in intervals of $1 \mathrm{eV}$ from $0 \mathrm{eV}$ up to $9 \mathrm{eV}$. For each material, the atomic positions and lattice parameters were fully relaxed until the required convergence criterion was satisfied. Calculated values were compared with experimental lattice parameters, magnetic moments, and band-gap values. On this basis, we determined a common $U_{\text {eff }}$ value of $8 \mathrm{eV}$ to give the closest representation of the properties of interest in all three copper oxides. Using the DFT $+U$ generated wave functions and relaxed geometries as starting points, additional hybrid DFT calculations were performed in two different ways. In the first approach, we allowed the geometry to fully relax, while the second approach included one static self-consistent calculation, which showed no significant differences in band structures, magnetic configurations, or lattice parameters. Frequency-dependent dielectric calculations were performed within the independent particle approximation, and electronic allowed or forbidden band gaps were determined from the dipole matrix element square at the $\Gamma$ point, $|M|^{2}$. $|M|$ contains dipole transition matrix elements between valence and conduction states. If $|M|^{2}<10^{-3} \mathrm{eV}^{2} / \AA^{2}$, the transition was considered to be dipole forbidden, otherwise it was viewed as dipole allowed [9]. Local-field effects have been neglected. The number of empty conduction states in the calculation was checked for convergence until the same values were obtained as resulted from density functional perturbation theory embedded in VASP. We noted that the dipole matrix elements do not depend on the employed XC functional, i.e., they retain the nature of transitions. To assess the PV absorbing potential of copper oxides we followed the selection metric of spectroscopic limited maximum efficiency (SLME) proposed by $\mathrm{Yu}$ and Zunger [44]. This concept allowed us to evaluate the PV conversion efficiency of copper oxides relying on two calculated values: absorption spectrum and band gap. SLME reproduces the SQ metric in the simple case of direct allowed band-gap materials. The elastic tensor was determined by performing finite lattice distortions (which allow the calculation of the Hessian matrix) and elastic constants were derived from the strain-stress relationship [45]. Spin-orbit coupling and excitonic effects were not included in this work.

\section{RESULTS AND DISCUSSION}

Copper (I) oxide is a p-type semiconductor with a highsymmetry cubic structure. It contains six atoms in the unit cell, four $\mathrm{Cu}$ atoms which form a face-centred sublattice, and two $\mathrm{O}$ atoms which constitute a body-centred sublattice (see Table I for a summary of crystallographic and geometric properties), as shown in Fig. 1. Copper (II) oxide crystalizes in a lower symmetry monoclinic structure. The unit cell consists of eight atoms, four $\mathrm{Cu}$ and four $\mathrm{O}$ atoms, where each copper atom is coordinated by four oxygen atoms in an approximately square planar configuration. Paramelaconite $\left(\mathrm{Cu}_{4} \mathrm{O}_{3}\right)$, a rare intermediate between $\mathrm{CuO}$ and $\mathrm{Cu}_{2} \mathrm{O}$, crystalizes in a 
TABLE I. Experimental values of structural parameters for $\mathrm{Cu}_{2} \mathrm{O}, \mathrm{Cu}_{4} \mathrm{O}_{3}$, and $\mathrm{CuO}$ taken from Refs. [22], [23], and [12], respectively.

\begin{tabular}{lccc}
\hline \hline & $\mathrm{Cu}_{2} \mathrm{O}$ & $\mathrm{Cu}_{4} \mathrm{O}_{3}$ & $\mathrm{CuO}(\mathrm{eV})$ \\
\hline & $a=4.269$ & $a=5.837$ & $a=4.683$ \\
Lattice constant $(\AA)$ & & $c=9.932$ & $b=3.422$ \\
& & & $c=5.128$ \\
Space group & $P n \overline{3} m(224)$ & $\mathrm{I}_{1} /$ amd $(141)$ & $C 2 / c(15)$ \\
Crystal structure & cubic & tetragonal & monoclinic \\
$\mathrm{Cu}-\mathrm{O}(\AA)$ & 1.849 & 1.870 & 1.960 \\
$\mathrm{O}-\mathrm{O}(\AA)$ & 3.698 & 2.560 & 2.620 \\
$\mathrm{Cu}-\mathrm{Cu}(\AA)$ & 3.019 & 2.920 & 2.900 \\
\hline \hline
\end{tabular}

tetragonal structure. The main difference between $\mathrm{CuO}$ and $\mathrm{Cu}_{4} \mathrm{O}_{3}$ is that the addition of oxygen to certain atomic sites converts a rod of $\mathrm{Cu}^{+}$into a rod of $\mathrm{Cu}^{2+}$ atoms. This causes a change in the topology of the magnetic lattice, making $\mathrm{Cu}_{4} \mathrm{O}_{3}$ antiferromagnetic (AFM) below $40 \mathrm{~K}$ with a magnetic moment localized on the $\mathrm{Cu}^{2+}$ atoms with a value of $0.46-0.66 \mu_{\mathrm{B}}$ [12]. Thus far, $\mathrm{Cu}_{4} \mathrm{O}_{3}$ has been synthesized as thin films, while it has still not been possible to completely eliminate the bulk form from mixture phases [46].

\section{A. Structural and mechanical properties}

We first modelled the copper-oxide structures by considering the bulk unit cell. Overall, the calculated lattice parameters reproduce experimental data very well, as shown at the top of Table II.

Elastic constants and elastic moduli are among the most fundamental parameters that provide information on the mechanical properties and behavior of materials. Depending on the lattice symmetry and point group, the number of inequivalent elastic coefficients for each oxide of copper differs: 3 for $\mathrm{Cu}_{2} \mathrm{O}, 13$ for $\mathrm{CuO}$, and 6 for $\mathrm{Cu}_{4} \mathrm{O}_{3}$. We have calculated the elastic constants and different elastic properties, e.g., the bulk modulus $(B)$, shear modulus $(G)$, effective Young's modulus $(E)$, and Poisson's ratio $(v)$, and summarized them in Tables II-V. To confirm values obtained from elastic constant calculations we have deduced the bulk modulus from a BirchMurnaghan equation of state (EOS) [49]. We note how calculated values fall within the experimental range of available
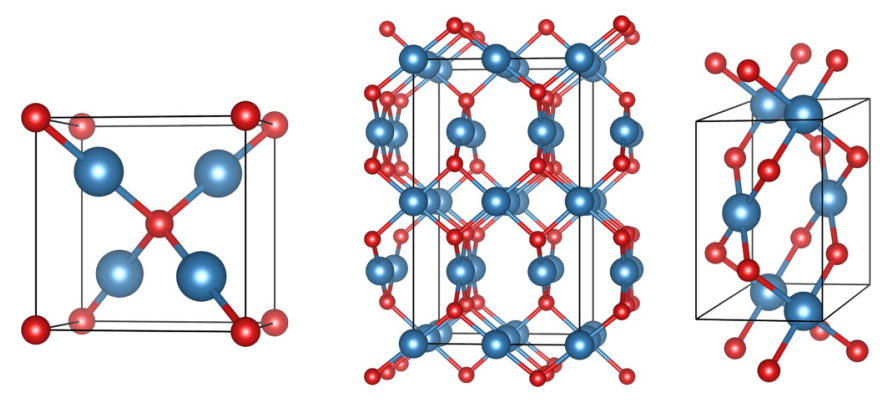

FIG. 1. Crystal structure of copper oxide compounds: (left) $\mathrm{Cu}_{2} \mathrm{O}$ (left), $\mathrm{Cu}_{4} \mathrm{O}_{3}$ (middle), and $\mathrm{CuO}$ (right). Blue coloured balls represent copper atoms, while red coloured balls represent oxygen atoms. data, confirming the high accuracy of the proposed modeling approach. Furthermore, compared to previous theoretical data, the elastic constants do not show negative values, indicating the stability and rigidness of bulk copper oxides.

We have also explored the stiffness of copper oxides through calculations of the microhardness parameter $(H)$ [47], which denotes the resistance of a physical object against compression of the contacting parts. Based on this characterization, only copper (II) oxide can be regarded as a hard material, with $\mathrm{Cu}_{4} \mathrm{O}_{3}$ falling closely behind (hard materials reach values of $H>10$ [51]). In addition, the equivalent Zener anisotropy factor $\left(A_{z}^{e q}\right)$ was examined in order to explore the anisotropy characteristics of the crystal. A factor that is close to 1 means that the material is fully isotropic, otherwise it is elastically anisotropic. Interestingly, the value for $\mathrm{Cu}_{2} \mathrm{O}$ is higher than the one for $\mathrm{CuO}$, indicating greater isotropy for the monoclinic structure (perhaps due to instability), with $\mathrm{Cu}_{4} \mathrm{O}_{3}$ being highly anisotropic.

\section{B. Electronic properties}

In contrast to its mechanical properties, the electronic properties of $\mathrm{Cu}_{2} \mathrm{O}$ are not correctly described with GGA, giving rise to a band-gap value of only $0.5 \mathrm{eV}$. An additional potential $\left(+U_{\text {eff }}\right)$ to GGA increases the value to $0.95 \mathrm{eV}$, which is still less than half of the experimental value (2.10-2.38 eV [40]). The cause of this discrepancy has been explained and reported extensively $[11,52,53]$. A major improvement was obtained using hybrid functionals, which led to the calculated values listed in Table VI (band structure shown in Fig. 2). Broadly speaking, the electronic structure calculations of bulk $\mathrm{Cu}_{2} \mathrm{O}$ show the typical features that have already been described $[4,9]$.

Calculations of the electronic dipole transition matrix provide insight into the nature of certain band transitions and rationalize the link to absorption properties. For $\mathrm{Cu}_{2} \mathrm{O}$, the first transition between the top of the valence band and the bottom of the conduction band is electric dipole forbidden, agreeing with previous observations [54-56]. Nevertheless, this transition is electric quadrupole allowed (and possibly magnetic dipole allowed, which has only been speculated upon so far) so that, linking it to the absorption spectra and dielectric function, it will give rise to a small, almost negligible absorption onset. The first allowed transitions are from the two top valence bands to the second conduction band with calculated energies of 2.478 and $2.485 \mathrm{eV}$, respectively. The nature and values of the transitions correspond very well with experimental data, as shown in Table VI. Furthermore, it should also be noted that no spin-orbit interaction was included in the calculations, which is known to affect the top of the valence band by an additional splitting of $0.15 \mathrm{eV}$ [9].

$\mathrm{CuO}$ is a strongly correlated system and standard DFT fails to predict its semiconducting ground state, obtaining a nonmagnetic (zero local magnetic moment) and metallic ground state instead [57-59]. However, by adding and tuning the $+U_{\text {eff }}$ value, the electronic and magnetic structure of cupric oxide is simulated accurately $[32,60]$. Surprisingly, adding a portion of the exact HF exchange does not seem to systematically improve the electronic structure calculations for $\mathrm{CuO}$ (band structure has been shown in Fig. 2). Heinemann 
TABLE II. Calculated values of structural and elastic properties for $\mathrm{Cu}_{2} \mathrm{O}, \mathrm{Cu}_{4} \mathrm{O}_{3}$, and $\mathrm{CuO}$ using DFT, DFT $+U(U=8 \mathrm{eV})$, and $\mathrm{HSE06}$. The shear and first bulk modulus were calculated using the Voigt-Reuss-Hill approximation [47], while an additional bulk modulus was calculated using the Birch-Murnaghan equation of state.

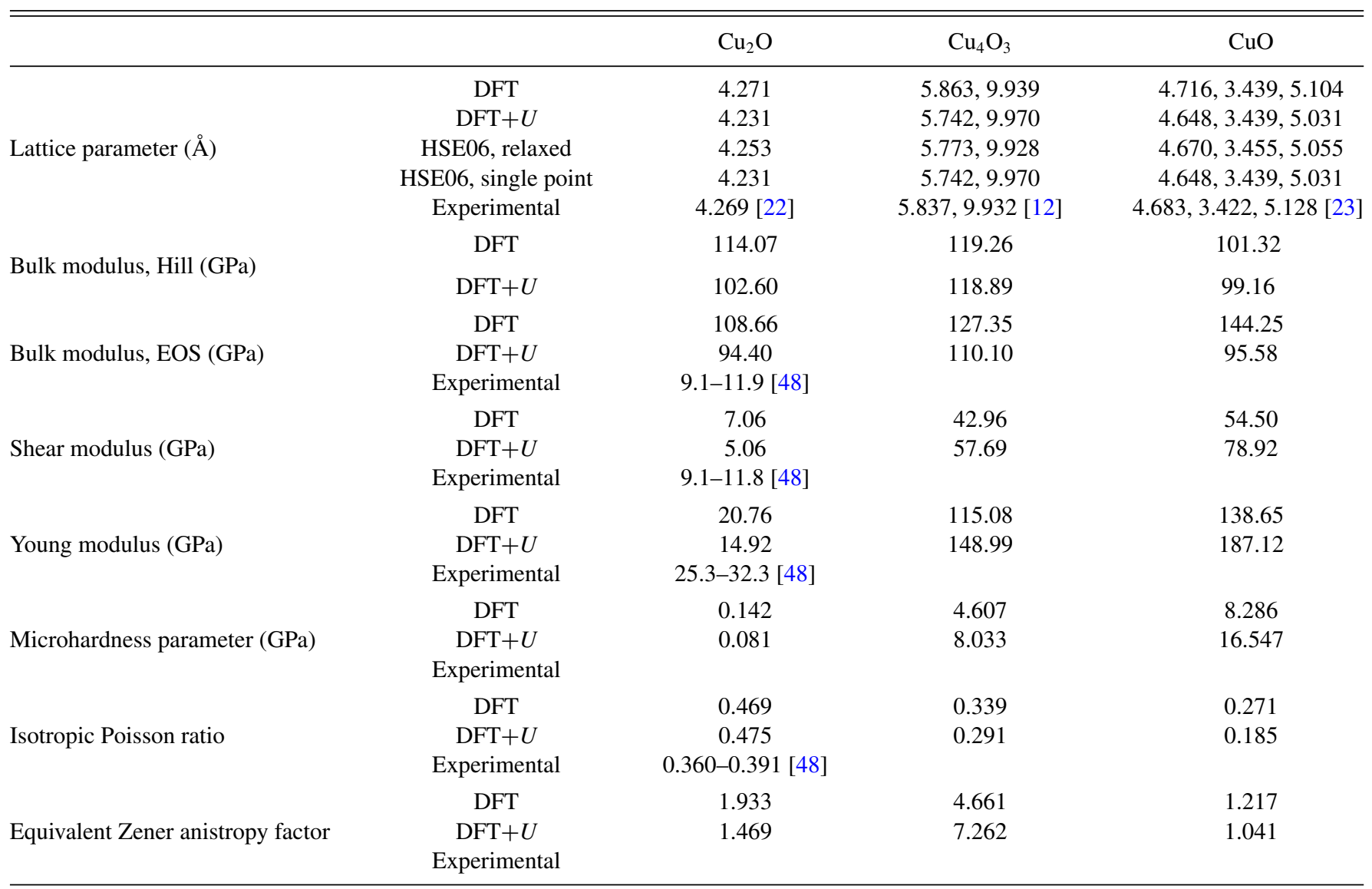

et al. [12] performed HSE06 calculations on top of LDA $+U$ and obtained a value of $2.74 \mathrm{eV}$ with an indirect nature of the band gap and AFM ordering. Our calculations employing hybrid functionals on top of GGA $+U$ gave a value of $2.102 \mathrm{eV}$ with the same indirect nature of the fundamental transition and a magnetic moment placed entirely on copper atoms with an individual value of $0.70 \mu_{\mathrm{B}}$ (agreeing well with the experimental value of $0.68 \mu_{\mathrm{B}}$ [34]). In addition, we calculated and compared the total energies of all the possible AFM ground-state configurations of $\mathrm{CuO}$ employing hybrid functionals. Surprisingly, band-gap values range from 2.102 to $2.718 \mathrm{eV}$, keeping the nature of the transition intact (Fig. 3). This indicates that not all AFM spin configurations are equally favorable and that $\mathrm{CuO}$ is sensitive to the initial calculation parameters.

However, while comparing those results, one must keep in mind that the experimental results are still neither conclusive

TABLE III. Calculated values of elastic constants for $\mathrm{Cu}_{2} \mathrm{O}$ using DFT, DFT $+U(U=8 \mathrm{eV})$.

\begin{tabular}{lccc}
\hline \hline & $c_{11}$ & $c_{12}$ & $c_{44}$ \\
\hline Expt. [50] & $116-126$ & $105-108$ & $11-13$ \\
DFT & 121.46 & 110.39 & 2.86 \\
DFT $+U$ & 106.23 & 100.79 & 3.99 \\
\hline \hline
\end{tabular}

about the value of the band gap nor its nature. Significant discrepancies in values (ranging from $1.0 \mathrm{eV}$ to almost $2.0 \mathrm{eV}$ ) could be attributed to the low symmetry and anisotropy of the crystal [61], strong temperature dependence of the band gap (due to strong electron-phonon coupling) [62], large temperature dependence of the absorption edge and Urbach tail (connected to the previous point) [63], and to the inadequacy of the simple Tauc expression to fit the experimental absorbance data [61].

Dipole transition matrix element calculations could clarify the picture for $\mathrm{CuO}$. These suggest that the nature of the transport gap is not only indirect, but also forbidden on the basis of the electric dipole. Furthermore, the next three transitions between the valence and conduction band (ordered by the lowest possible energy) indicate dipole forbidden character. The first allowed transition occurs at an energy difference bigger than $4 \mathrm{eV}$ and only in certain directions within the

TABLE IV. Calculated values of elastic constants for $\mathrm{Cu}_{4} \mathrm{O}_{3}$ using DFT, DFT $+U(U=8 \mathrm{eV})$. No experimental work is, to the best of our knowledge, available to compare with.

\begin{tabular}{lcccccc}
\hline \hline & $c_{11}$ & $c_{12}$ & $c_{44}$ & $c_{13}$ & $c_{33}$ & $c_{66}$ \\
\hline DFT & 156.96 & 64.14 & 9.96 & 113.52 & 227.51 & 7.78 \\
DFT $+U$ & 186.76 & 44.01 & 9.82 & 101.38 & 250.07 & 3.92 \\
\hline
\end{tabular}


TABLE V. Calculated values of elastic constants for $\mathrm{CuO}$ using DFT, DFT $+U(U=8 \mathrm{eV})$.

\begin{tabular}{lccccccc}
\hline \hline & $c_{11}$ & $c_{12}$ & $c_{44}$ & $c_{13}$ & $c_{33}$ & $c_{66}$ & \\
\hline Ref. [50] & 196.41 & 122.63 & 20.28 & 114.64 & 293.70 & 56.44 & \\
DFT & 243.28 & 132.03 & 45.70 & 151.88 & 277.56 & 63.50 & \\
DFT+U & 258.19 & 139.52 & 56.99 & 118.93 & 312.80 & 106.67 & \\
& $c_{22}$ & $c_{55}$ & $c_{15}$ & $c_{23}$ & $c_{25}$ & $c_{35}$ & $c_{46}$ \\
Ref. [50] & 125.01 & 30.07 & -26.99 & 96.89 & -27.85 & -48.94 & -1.15 \\
DFT & 96.26 & 13.59 & 0.00 & 87.71 & 0.00 & 0.00 & 0.00 \\
DFT+U & 109.83 & 56.99 & 0.00 & 57.26 & 0.00 & 0.00 & 0.00 \\
\hline \hline
\end{tabular}

crystal. This information could serve as a starting point for new and accurate experimental work, but also for theoretical work incorporating spin-orbit coupling effects, which could affect those values and induce possible band splitting.

Unlike the case of $\mathrm{CuO}$, basic DFT reproduces a small band-gap value for $\mathrm{Cu}_{4} \mathrm{O}_{3}$ and results in fully nonmagnetic behavior. By employing GGA $+U$ methodology the value of the gap increases to $1.558 \mathrm{eV}$, together with a recovery of the AFM ordering and magnetic moment of $0.71 \mu_{\mathrm{B}}$. Hybrid functional calculations show a dipole forbidden indirect band transition between the valence and conduction band, with a band-gap value of $2.370 \mathrm{eV}$ and magnetic moment of $0.68 \mu_{\mathrm{B}}$, as is shown in Fig. 2 (agreeing well with the experimental value of $\left.0.46-0.66 \mu_{\mathrm{B}}[12]\right)$. However, as Heinemann et al. [12] pointed out, experimental values for $\mathrm{Cu}_{4} \mathrm{O}_{3}$ are still inconclusive regarding both the value and the nature of the band transition.

\section{Optical properties}

The calculated real and imaginary parts of the dielectric functions of $\mathrm{Cu}_{2} \mathrm{O}, \mathrm{CuO}$, and $\mathrm{Cu}_{4} \mathrm{O}_{3}$, together with their corresponding absorption coefficients obtained using HSE06, are shown in Figs. 4 and 5. In the calculated absorption spectra of $\mathrm{Cu}_{2} \mathrm{O}$, the two aforementioned allowed transitions can be observed at around $500 \mathrm{~nm} \approx 2.5 \mathrm{eV}$. Hybrid functionals tend to give roughly the right positions of the peaks but slightly underestimate their intensity. The imaginary part of the dielectric function shows similar results for the positions of the transition energies.

Within the experimental data of the imaginary part of the dielectric function of $\mathrm{CuO}$, Meyer et al. [9] were able to identify multiple positions of possible interband transitions. Our dipole matrix calculations explain why it is difficult to
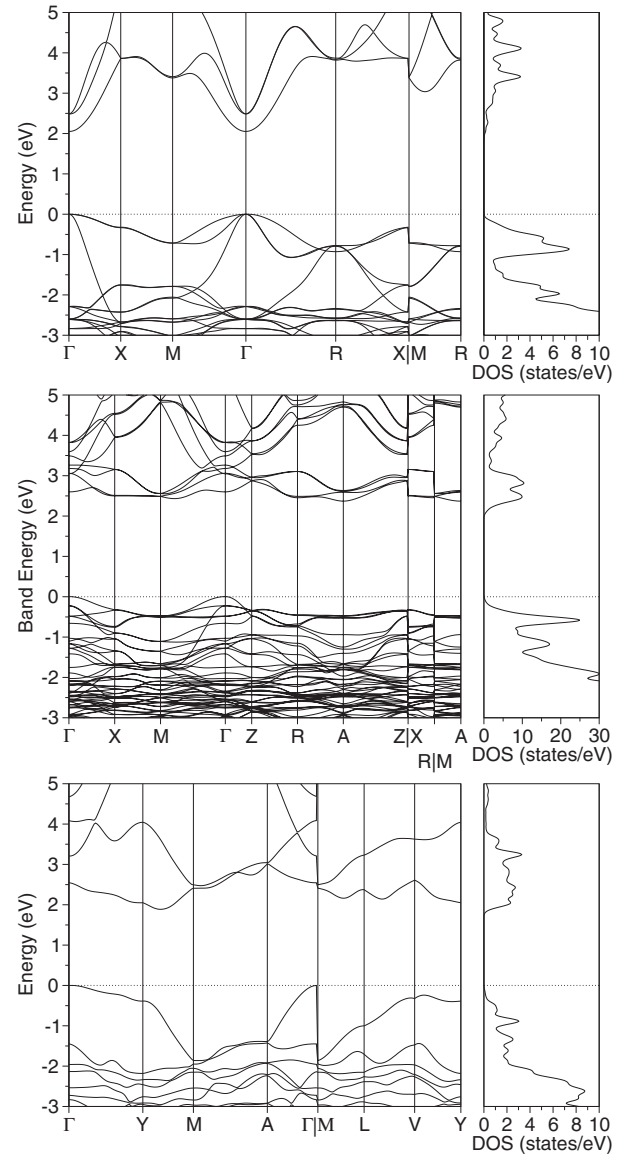

FIG. 2. Band-structure diagrams of $\mathrm{Cu}_{2} \mathrm{O}, \mathrm{Cu}_{4} \mathrm{O}_{3}$, and $\mathrm{CuO}$. The valence-band maximum has been set to zero.

identify those transitions within the dielectric function or by looking at the shape of the absorption coefficient curve. Unlike $\mathrm{Cu}_{2} \mathrm{O}, \mathrm{CuO}$ seems to have a more complex band structure and transition occurrence between certain bands, where a dipole forbidden nature could be responsible for hardly visible interband transitions. Looking closer at the absorption coefficient curve between 2 and $3 \mathrm{eV}$ gives an idea when the absorption of photons starts to take place, but the extremely low intensity and smeared peaks are not conclusive about a precise onset of absorption.

Calculations of optical properties for $\mathrm{Cu}_{4} \mathrm{O}_{3}$ suggest low absorption until the energy of the incident photon reaches $3.250 \mathrm{eV}$ (around $380 \mathrm{~nm}$ ). One additional stronger peak can be observed at around $3.600 \mathrm{eV}$ (around $345 \mathrm{~nm}$ ) which

TABLE VI. Calculated band-gap energies of $\mathrm{Cu}_{2} \mathrm{O}, \mathrm{Cu}_{4} \mathrm{O}_{3}$, and $\mathrm{CuO}$ compared to experimental values. Also, the estimated nature of the transition together with the type of the band gap are enlisted.

\begin{tabular}{lcccccc}
\hline \hline Compound & Type of transition & Nature of transition & DFT (eV) & DFT $+U(\mathrm{eV})$ & HSE06 (eV) & Experimental value (eV) \\
\hline $\mathrm{Cu}_{2} \mathrm{O}$ & Direct & Dipole forbidden & 0.535 & 0.945 & 2.052 & $2.10-2.38[64]$ \\
$\mathrm{Cu}_{4} \mathrm{O}_{3}$ & Indirect & & & & 2.370 & $1.34-1.50[12], 1.75[65]$ \\
& Direct & Dipole forbidden & 0.333 & 1.558 & 2.620 & \\
$\mathrm{CuO}$ & Indirect & & & 1.485 & 2.102 & $1.0-1.9[9,23,66]$ \\
& Direct & Dipole forbidden & 0.607 & 1.916 & 2.578 & \\
\hline \hline
\end{tabular}



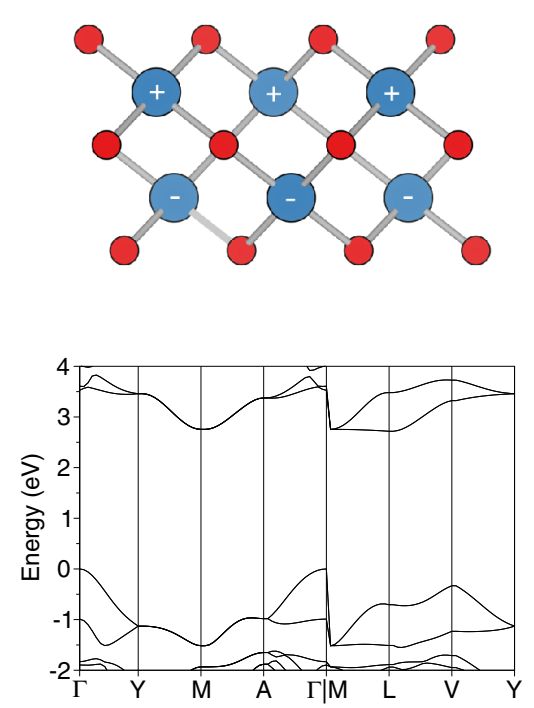
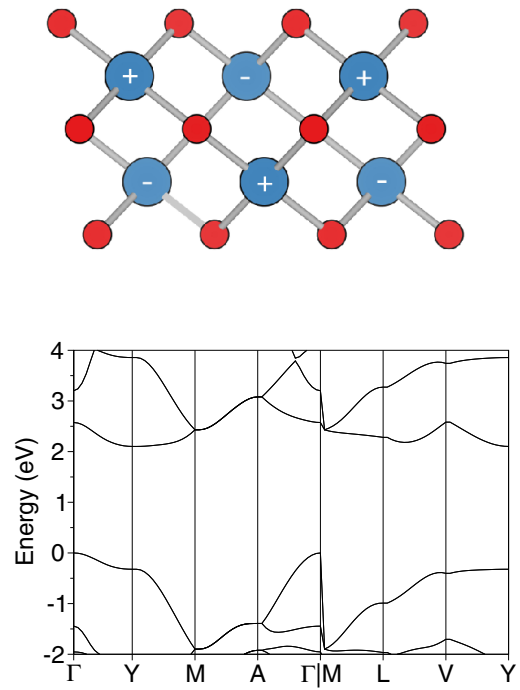
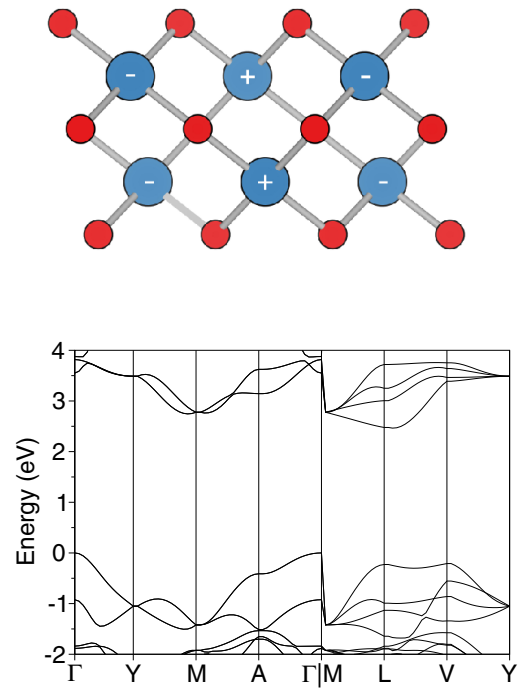

FIG. 3. Calculated band structures using HSE06 functional for $\mathrm{CuO}$ in three different ground-state antiferromagnetic configurations.

could be assigned to a possible interband transition, but to the best of our knowledge, experimental data are lacking to confirm this suggestion. Other transitions cannot be observed as the absorption curve shows hardly any peaks in the relevant visible spectrum.

\section{Photovoltaic absorber efficiency}

By combining the nature of the elementary transitions between valence and conduction bands with their dipole allowance and accompanying absorption spectra, we have extended the classic SQ limit for copper oxides to the SLME metric. This approach classifies copper oxides into different groups of optical materials, according to the order of dipole allowed and forbidden transitions (more details on the division scheme can be found in Yu and Zunger [44]). As mentioned before, the lowest energy transition of $\mathrm{Cu}_{2} \mathrm{O}$ is direct dipole forbidden, followed by a direct allowed transition. For $\mathrm{Cu}_{4} \mathrm{O}_{3}$ and $\mathrm{CuO}$ the first transitions are indirect dipole forbidden as shown in Fig. 6. In the case of $\mathrm{Cu}_{4} \mathrm{O}_{3}$, the following transition is an indirect, but dipole allowed, transition, while for $\mathrm{CuO}$ we note only forbidden transitions within the visible part of the electromagnetic spectrum. SLME calculations give absorbing efficiencies based upon those considerations.

The calculated dependence of SLME on the film thickness for copper oxides is shown in Fig. 7. Choosing a reasonable reference thin-film thickness of $0.5 \mu \mathrm{m}$ we obtain $0.54 \%$ and $1.40 \%$ efficiency for $\mathrm{Cu}_{2} \mathrm{O}$ and $\mathrm{Cu}_{4} \mathrm{O}_{3}$, respectively. This corresponds well with experimentally observed PCE values, which take values of $1-2 \%$ for $\mathrm{Cu}_{2} \mathrm{O}$ and $\mathrm{CuO}[4,9,68]$. For $\mathrm{Cu}_{4} \mathrm{O}_{3}$ no experimental PCE values have been obtained, to the best of our knowledge. It is also worth mentioning that increasing the thickness leads to a plateau in efficiency. In other words, $\mathrm{Cu}_{2} \mathrm{O}$ single-crystal compounds cannot exceed conversion efficiencies of $1 \%$ without further modification. For $\mathrm{Cu}_{4} \mathrm{O}_{3}$, this threshold is slightly higher, reaching 2-3\%. As noted, $\mathrm{CuO}$ does not appear in the analysis due to negligible efficiency values, attributed to low absorption onsets
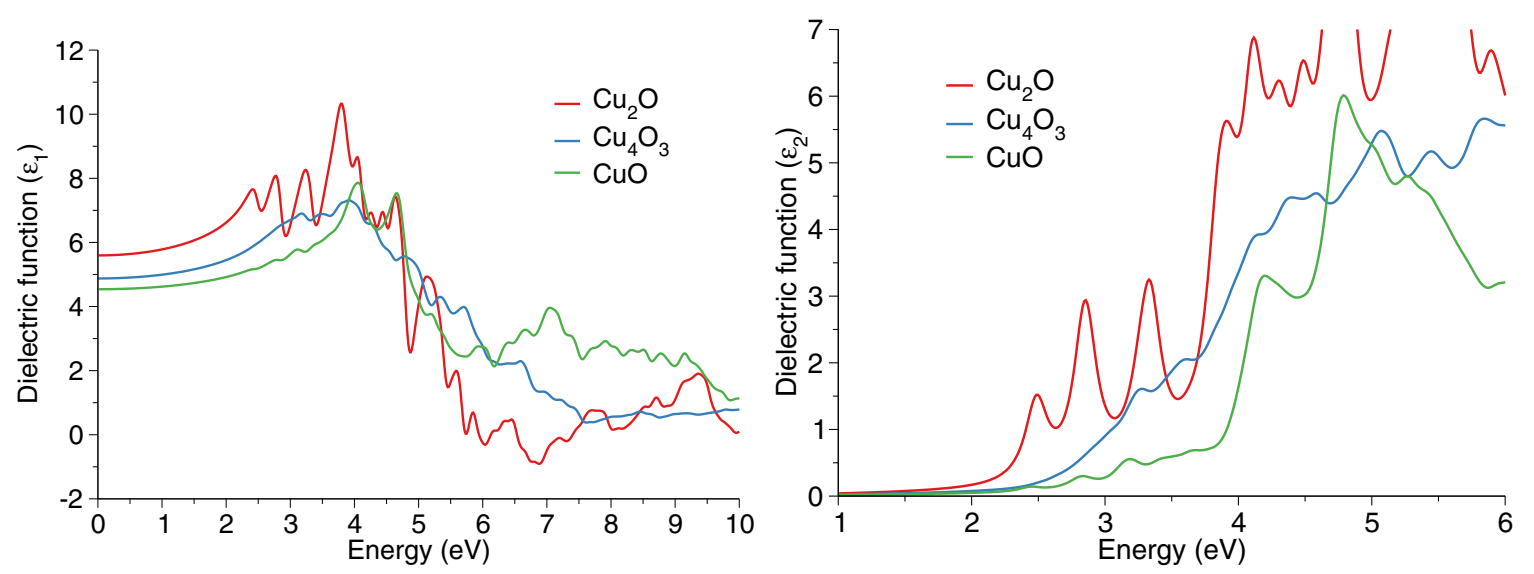

FIG. 4. Calculated real part of the dielectric function $\left(\varepsilon_{1}\right.$, left $)$ and imaginary part of the dielectric function $\left(\varepsilon_{2}\right.$, right $)$ for $\mathrm{Cu}_{2} \mathrm{O}, \mathrm{Cu}_{4} \mathrm{O}_{3}$, and $\mathrm{CuO}$ employing the HSE06 functional. 


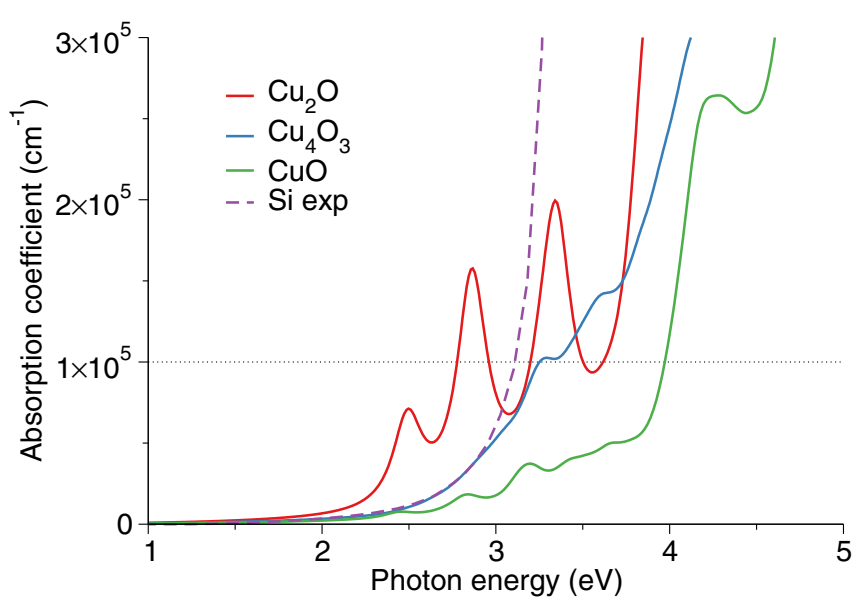

FIG. 5. Calculated absorption coefficient for $\mathrm{Cu}_{2} \mathrm{O}, \mathrm{Cu}_{4} \mathrm{O}_{3}$, and $\mathrm{CuO}$ using the HSE06 functional averaged over Cartesian coordinates. For comparison, the silicon experimental value has been shown with the purple dashed line [67].

and a clear majority of electrically dipole forbidden transitions that govern weak interactions between the incoming sunlight and valence electrons. In addition, photons that get absorbed by $\mathrm{CuO}$ are not efficiently converted due to significant nonradiative recombination processes inside the material. This correlates well with experimentally observed charge recombination processes preventing large-scale application of CuO-based solar cells $[69,70]$. This is attributed to the large radiative fraction (order of $10^{-40}$ ) which is used to calculate the reverse saturation current, imposing a significant influence on the open circuit voltage of the operating device. If we model $\mathrm{CuO}$ as a direct gap material, the maximum efficiency obtained does not exceed 2-3\%. This implies that even if the nonradiative recombination processes are neglected, the absorption of $\mathrm{CuO}$ is very low and does not produce favorable output voltages.

These findings suggest that single-crystal copper oxides cannot be efficiently employed as solar-collecting materials.
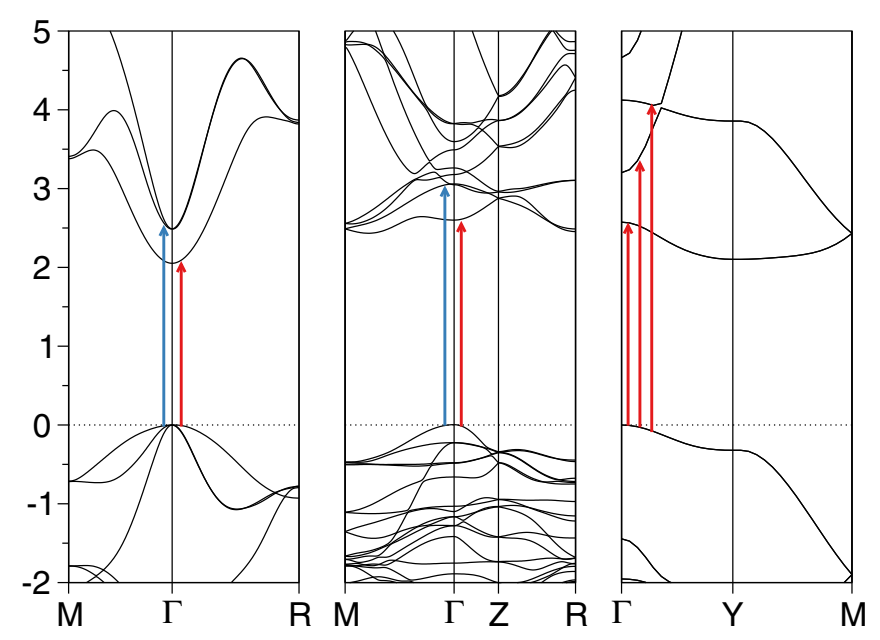

FIG. 6. Energy-band structure diagram of $\mathrm{Cu}_{2} \mathrm{O}, \mathrm{Cu}_{4} \mathrm{O}_{3}$, and $\mathrm{CuO}$ (from left to right) showing dipole allowed (blue arrow) and dipole forbidden (red arrow) transitions between the valence and conduction band.

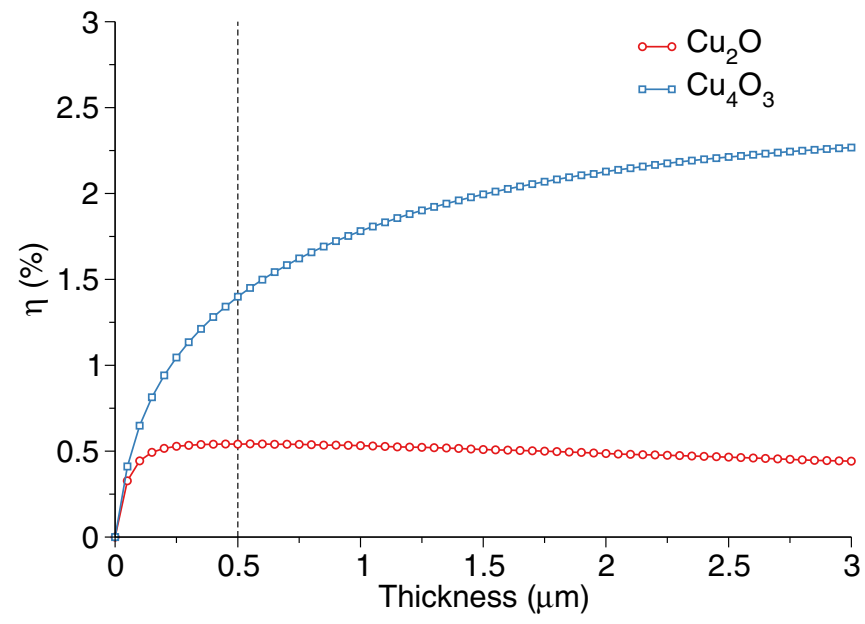

FIG. 7. Calculated spectroscopic limited maximum efficiency (SLME) plotted as a function of the film thickness.

However, modifying the material's inner properties could lead to notable improvements. I.e., introducing extrinsic dopants could allow one to tune the Fermi level and create defect states situated within the band gap. Photon-induced transitions from defect states can lead to enhanced photocurrent generation through subband-gap absorption. This has been already demonstrated by electrochemical doping in $n$-type $\mathrm{Cu}_{2} \mathrm{O}$ with chlorine [71]. It improved the conductivity of $\mathrm{Cu}_{2} \mathrm{O}$ and hence, the overall efficiency of an intrinsic $p-n$ junction of $\mathrm{Cu}_{2} \mathrm{O}$. Also, defect states can cause carrier trapping or additional nonradiative recombination, depending on the position of the defect state relative to the valence or conduction band. First-principle calculations can explore unreported promising materials or provide insights into defect energetics without the need of previous synthesis.

Another possibility is the combination of copper oxides with other materials to create tandem cells. Improvement has been demonstrated, i.e., by creating heterojunction solar cells between $\mathrm{AZO}, \mathrm{Ga}_{2} \mathrm{O}_{3}$, and $\mathrm{Cu}_{2} \mathrm{O}$. This has led to an increase in efficiency to almost 5.5\% [6]. If we compare this efficiency to the almost negligible conversion as a single cell, it offers suggestions for new designs. However, while forming junctions with copper oxides, the control of the interface properties is crucial in order to avoid mixture phases. Again, firstprinciple calculations can provide useful information which include Schottky barrier heights of interfaces, band offsets, or band positioning.

\section{CONCLUSION}

We have modelled the photovoltaic efficiency of copper oxides using density functional theory. Our DFT $+U$ and HSE06 calculations have proven to be a computationally feasible and accurate approach to determine the electronic, magnetic, and optical properties. $\mathrm{Cu}_{2} \mathrm{O}, \mathrm{Cu}_{4} \mathrm{O}_{3}$, and $\mathrm{CuO}$ are shown to be semiconductors with different types of fundamental band-gap transition. Moreover, we have reported dipole transition matrix calculations which allowed us to characterize certain transitions from the valence to the conduction band and to assign them to peaks in the dielectric function and 
absorption spectrum. Finally, we have extended the usual SQ limit into the SLME metric, which has allowed us to explain why bulk single-crystal copper-oxides solar cells have low photovoltaic conversion efficiencies, as is seen experimentally. Future calculations could incorporate phonon-assisted interband transitions and absorption spectra into the SLME metric (or equivalent) in order to obtain even better descriptors and accurate prediction.

Information on the data underpinning the results presented here, including how to access them, can be found in the Cardiff University data catalog [72].

\section{ACKNOWLEDGMENTS}

We acknowledge the Cardiff University School of Chemistry for a Ph.D. studentship for A.Ž. and the Royal Society DfID Africa program for funding. This work was performed using the computational facilities of the Advanced Research Computing @ Cardiff (ARCCA) Division, Cardiff University. Via our membership of the UK's HPC Materials Chemistry Consortium, which is funded by EPSRC (EP/L000202), this work made use of the ARCHER facility, the UK's national high-performance computing service, which is funded by the Office of Science and Technology through EPSRC's High End Computing Programme.
[1] K. H. L. Zhang, K. Xi, M. G. Blamire, and R. G. Egdell, P-type transparent conducting oxides, J. Phys.: Condens. Matter 28, 383002 (2016).

[2] R. Wick and S. D. Tilley, Photovoltaic and photoelectrochemical solar energy conversion with $\mathrm{Cu}_{2} \mathrm{O}$, J. Phys. Chem. C 119, 26243 (2015); L. Vinet and A. Zhedanov, A 'missing' family of classical orthogonal polynomials, J. Phys. A: Math. Theor. 44 085201 (2011).

[3] Y. Peng, Z. Zhang, T. Viet Pham, Y. Zhao, P. Wu, and J. Wang, Density functional theory analysis of dopants in cupric oxide, J. Appl. Phys. 111, 103708 (2012).

[4] T. Wong, S. Zhuk, S. Masudy-Panah, and G. Dalapati, Current status and future prospects of copper oxide heterojunction solar cells, Materials (Basel) 9, 271 (2016).

[5] A. Y. Anderson, Y. Bouhadana, H. N. Barad, B. Kupfer, E. Rosh-Hodesh, H. Aviv, Y. R. Tischler, S. Rühle, and A. Zaban, Quantum efficiency and bandgap analysis for combinatorial photovoltaics: Sorting activity of $\mathrm{Cu}-\mathrm{O}$ compounds in all-oxide device libraries, ACS Comb. Sci. 16, 53 (2014).

[6] T. Minami, Y. Nishi, and T. Miyata, High-efficiency $\mathrm{Cu}_{2} \mathrm{O}-$ based heterojunction solar cells fabricated using a $\mathrm{Ga}_{2} \mathrm{O}_{3}$ thin film as N-Type layer, Appl. Phys. Exp. 6, 044101 (2013).

[7] W. Shockley and H. J. Queisser, Detailed balance limit of efficiency of $p n$ junction solar cells, J. Appl. Phys. 32, 510 (1961).

[8] C. Jayathilaka, V. Kapaklis, W. Siripala, and S. Jayanetti, Improved efficiency of electrodeposited $\mathrm{p}-\mathrm{CuO} / \mathrm{n}-\mathrm{Cu}_{2} \mathrm{O}$ heterojunction solar cell, Appl. Phys. Exp. 8, 065503 (2015).

[9] B. K. Meyer, A. Polity, D. Reppin, M. Becker, P. Hering, P. J. Klar, T. Sander, C. Reindl, J. Benz, M. Eickhoff, C. Heiliger, M. Heinemann, J. Bläsing, A. Krost, S. Shokovets, C. Müller, and C. Ronning, Binary copper oxide semiconductors: From materials towards devices, Phys. Status Solidi 249, 1487 (2012).

[10] L. K. Dash, F. Bruneval, V. Trinité, N. Vast, and L. Reining, Electronic excitations: Ab initio calculations of electronic spectra and application to zirconia $\mathrm{ZrO}_{2}$, titania $\mathrm{TiO}_{2}$ and cuprous oxide $\mathrm{Cu}_{2} \mathrm{O}$, Comput. Mater. Sci. 38, 482 (2007).

[11] M. Nolan and S. D. Elliott, The p-type conduction mechanism in $\mathrm{Cu}_{2} \mathrm{O}$ : A first principles study, Phys. Chem. Chem. Phys. 8, 5350 (2006).

[12] M. Heinemann, B. Eifert, and C. Heiliger, Band structure and phase stability of the copper oxides $\mathrm{Cu}_{2} \mathrm{O}, \mathrm{CuO}$, and $\mathrm{Cu}_{4} \mathrm{O}_{3}$, Phys. Rev. B 87, 115111 (2013).

[13] H. Wu, N. Zhang, Z. Cao, H. Wang, and S. Hong, The adsorption of $\mathrm{CO}_{2}, \mathrm{H}_{2} \mathrm{CO}_{3}, \mathrm{HCO}_{3}^{-}$and $\mathrm{CO}_{3}{ }^{2-}$ on $\mathrm{Cu}_{2} \mathrm{O}$ (111) surface: Firstprinciples study, Int. J. Quantum Chem. 112, 2532 (2012).

[14] X.-G. Yan, L. Xu, W.-Q. Huang, G.-F. Huang, Z.-M. Yang, S.-Q. Zhan, and J.-P. Long, Theoretical insight into the electronic and photocatalytic properties of $\mathrm{Cu}_{2} \mathrm{O}$ from a hybrid density functional theory, Mater. Sci. Semicond. Process. 23, 34 (2014).

[15] L. Hedin, New method for calculating the one-particle green's function with application to the electron-gas problem, Phys. Rev. 139, A796 (1965).

[16] L. Hedin and S. Lundqvist, in Solid State Physics (Academic Press, New York, 1970), Vol. 23, pp. 1-181.

[17] F. Bruneval, N. Vast, L. Reining, M. Izquierdo, F. Sirotti, and N. Barrett, Exchange and Correlation Effects in Electronic Excitations of $\mathrm{Cu}_{2} \mathrm{O}$, Phys. Rev. Lett. 97, 267601 (2006).

[18] S. Lany, Band-structure calculations for the $3 d$ transition metal oxides in GW, Phys. Rev. B 87, 085112 (2013).

[19] Y. Wang, S. Lany, J. Ghanbaja, Y. Fagot-Revurat, Y. P. Chen, F. Soldera, D. Horwat, F. Mücklich, and J. F. Pierson, Electronic structures of $\mathrm{Cu}_{2} \mathrm{O}, \mathrm{Cu}_{4} \mathrm{O}_{3}$, and $\mathrm{CuO}$ : A joint experimental and theoretical study, Phys. Rev. B 94, 245418 (2016).

[20] B. J. Morgan, D. O. Scanlon, and G. W. Watson, The use of the " $+U$ " Correction in describing defect states at metal oxide surfaces: Oxygen vacancies on $\mathrm{CeO}_{2}$ and $\mathrm{TiO}_{2}$, and Li-doping of MgO, e-Journal Surf. Sci. Nanotechnol. 7, 389 (2009).

[21] C. Rödl, F. Sottile, and L. Reining, Quasiparticle excitations in the photoemission spectrum of $\mathrm{CuO}$ from first principles: $\mathrm{A} G W$ study, Phys. Rev. B 91, 045102 (2015).

[22] N. N. Greenwood and A. Earnshaw, Copper, silver and gold, in Chemistry of the Elements (Elsevier, 1997), pp. 1173-1200.

[23] S. C. Ray, Preparation of copper oxide thin film by the solgellike dip technique and study of their structural and optical properties, Sol. Energy Mater. Sol. Cells 68, 307 (2001).

[24] G. Kresse and J. Furthmüller, Efficiency of ab-initio total energy calculations for metals and semiconductors using a plane-wave basis set, Comput. Mater. Sci. 6, 15 (1996).

[25] G. Kresse and J. Furthmüller, Efficient iterative schemes for $a b$ initio total-energy calculations using a plane-wave basis set, Phys. Rev. B 54, 11169 (1996).

[26] P. E. Blöchl, Projector augmented-wave method, Phys. Rev. B 50, 17953 (1994); J. Lehtomäki, I. Makkonen, M. A. Caro, A. Harju, and O. Lopez-Acevedo, Orbital-free density 
functional theory implementation with the projector augmented-wave method, J. Chem. Phys. 141, 234102 (2014).

[27] G. Kresse and D. Joubert, From ultrasoft pseudopotentials to the projector augmented-wave method, Phys. Rev. B 59, 1758 (1999).

[28] J. P. Perdew, K. Burke, and M. Ernzerhof, Generalized Gradient Approximation Made Simple, Phys. Rev. Lett. 77, 3865 (1996).

[29] S. L. Dudarev, G. A. Botton, S. Y. Savrasov, C. J. Humphreys, and A. P. Sutton, Electron-energy-loss spectra and the structural stability of nickel oxide: An LSDA+U study, Phys. Rev. B 57, 1505 (1998).

[30] V. I. Anisimov, J. Zaanen, and O. K. Andersen, Band theory and Mott insulators: Hubbard $U$ instead of Stoner I, Phys. Rev. B 44, 943 (1991).

[31] V. I. Anisimov, I. V. Solovyev, M. A. Korotin, M. T. Czyżyk, and G. A. Sawatzky, Density-functional theory and NiO photoemission spectra, Phys. Rev. B 48, 16929 (1993).

[32] V. I. Anisimov, F. Aryasetiawan, and A. I. Lichtenstein, Firstprinciples calculations of the electronic structure and spectra of strongly correlated systems: The LDA $+U$ method, J. Phys.: Condens. Matter 9, 767 (1997).

[33] J. Heyd, G. E. Scuseria, and M. Ernzerhof, Hybrid functionals based on a screened Coulomb potential, J. Chem. Phys. 118, 8207 (2003).

[34] J. Heyd and G. E. Scuseria, Efficient hybrid density functional calculations in solids: Assessment of the Heyd-ScuseriaErnzerhof screened Coulomb hybrid functional, J. Chem. Phys. 121, 1187 (2004).

[35] J. Heyd, G. E. Scuseria, and M. Ernzerhof, Erratum: "Hybrid functionals based on a screened Coulomb potential" [J. Chem. Phys. 118, 8207 (2003)], J. Chem. Phys. 124, 219906 (2006).

[36] H. J. Monkhorst and J. D. Pack, Special points for Brillouinzone integrations, Phys. Rev. B 13, 5188 (1976).

[37] S. Grimme, J. Antony, S. Ehrlich, and H. Krieg, A consistent and accurate $a b$ initio parametrization of density functional dispersion correction (DFT-D) for the 94 elements H-Pu, J. Chem. Phys. 132, 154104 (2010).

[38] M. I. Aroyo, J. M. Perez-Mato, C. Capillas, E. Kroumova, S. Ivantchev, G. Madariaga, A. Kirov, and H. Wondratschek, Bilbao crystallographic server: I. Databases and crystallographic computing programs, Z. Kristallogr. - Cryst. Mater. 221, 15 (2006).

[39] M. I. Aroyo, A. Kirov, C. Capillas, J. M. Perez-Mato, and H. Wondratschek, Bilbao crystallographic server. II. Representations of crystallographic point groups and space groups, Acta Crystallogr., Sect. A: Found. Crystallogr. 62, 115 (2006).

[40] M. I. Aroyo, J. M. Perez-Mato, D. Orobengoa, E. Tasci, G. De La Flor, and A. Kirov, Crystallography online: Bilbao crystallographic server, Bulg. Chem. Commun. 43, 183 (2011).

[41] A. A. Mostofi, J. R. Yates, G. Pizzi, Y.-S. Lee, I. Souza, D. Vanderbilt, and N. Marzari, An updated version of wannier90: A tool for obtaining maximally-localised Wannier functions, Comput. Phys. Commun. 185, 2309 (2014); A. A. Mostofi, J. R. Yates, Y.-S. Lee, I. Souza, D. Vanderbilt, and N. Marzari, wannier90: A tool for obtaining maximally-localised Wannier functions, 178, 685 (2008).

[42] K. Momma and F. Izumi, VESTA 3 for three-dimensional visualization of crystal, volumetric and morphology data, J. Appl. Crystallogr. 44, 1272 (2011).
[43] J. B. Forsyth, P. J. Brown, and B. M. Wanklyn, Magnetism in cupric oxide, J. Phys. C: Solid State Phys. 21, 2917 (1988).

[44] L. Yu and A. Zunger, Identification of Potential Photovoltaic Absorbers Based on First-Principles Spectroscopic Screening of Materials, Phys. Rev. Lett. 108, 068701 (2012).

[45] Y. Le Page and P. Saxe, Symmetry-general least-squares extraction of elastic coefficients from ab initio total energy calculations, Phys. Rev. B 63, 174103 (2001).

[46] L. Pinsard-Gaudart, J. Rodríguez-Carvajal, A. Gukasov, and P. Monod, Magnetic properties of paramelaconite $\left(\mathrm{Cu}_{4} \mathrm{O}_{3}\right)$ : A pyrochlore lattice with $S=\frac{1}{2}$, Phys. Rev. B 69, 104408 (2004).

[47] Y.-Y. Yu, W.-C. Chien, and Y.-J. Wang, Copper oxide hole transport materials for heterojunction solar cell applications, Thin Solid Films 618, 134 (2016).

[48] V. Belomestnykh and E. Soboleva, Behavior of poisson's ratio in the crystal $\mathrm{Cu}_{2} \mathrm{O}$, Appl. Mech. Mater. 682, 170 (2014).

[49] F. Birch, Finite elastic strain of cubic crystals, Phys. Rev. 71, 809 (1947).

[50] N. N. Liu, J. L. Sun, and D. Wu, Elastic constants and thermodynamic properties of $\mathrm{Cu}, \mathrm{Cu}_{2} \mathrm{O}$ and $\mathrm{CuO}$ from first-principles calculations, Adv. Mater. Res. 335-336, 328 (2011).

[51] M. M. Yovanovich, in 44th AIAA Aerospace Science Meeting and Exhibit (American Institute of Aeronautics and Astronautics, Viriginia, 2006), pp. 1-28.

[52] D. O. Scanlon, B. J. Morgan, G. W. Watson, and A. Walsh, Acceptor Levels in $p$-Type $\mathrm{Cu}_{2} \mathrm{O}$ : Rationalizing Theory and Experiment, Phys. Rev. Lett. 103, 096405 (2009).

[53] D. O. Scanlon, B. J. Morgan, and G. W. Watson, Modeling the polaronic nature of $p$-type defects in $\mathrm{Cu}_{2} \mathrm{O}$ : The failure of GGA and GGA $+U$, J. Chem. Phys. 131, 124703 (2009).

[54] F. Biccari, Defects and Doping in $\mathrm{Cu}_{2} \mathrm{O}$, Ph.D. thesis, University of Rome, Sapienza, 2009.

[55] F. Schweiner, J. Main, G. Wunner, and C. Uihlein, Even exciton series in $\mathrm{Cu}_{2} \mathrm{O}$, Phys. Rev. B 95, 195201 (2017).

[56] J. Thewes, J. Heckötter, T. Kazimierczuk, M. Aßmann, D. Fröhlich, M. Bayer, M. A. Semina, and M. M. Glazov, Observation of High Angular Momentum Excitons in Cuprous Oxide, Phys. Rev. Lett. 115, 027402 (2015).

[57] D. Wu, Q. Zhang, and M. Tao, LSDA+U study of cupric oxide: Electronic structure and native point defects, Phys. Rev. B 73, 235206 (2006)

[58] H. Fan, B. Zou, Y. Liu, and S. Xie, Size effect on the electronphonon coupling in $\mathrm{CuO}$ nanocrystals, Nanotechnology 17, 1099 (2006).

[59] U. Köbler and T. Chattopadhyay, On the magnetic anisotropy of CuO, Z. Phys. B: Condens. Matter 82, 383 (1991).

[60] C. E. Ekuma, V. Anisimov, J. Moreno, and M. Jarrell, Electronic structure and spectra of CuO, Eur. Phys. J. B 87, 23 (2014).

[61] K. Ito, Y. Matsuura, T. Nakazawa, and H. Takenouchi, Photovoltaic effect in monoclinic $\mathrm{ZnP}_{2}$, Jpn. J. Appl. Phys. 20, 109 (1981).

[62] S. Huotari, L. Simonelli, C. J. Sahle, M. M. Sala, R. Verbeni, and G. Monaco, Temperature dependence of crystal field excitations in CuO, J. Phys.: Condens. Matter 26, 165501 (2014).

[63] F. Marabelli, G. B. Parravicini, and F. Salghetti-Drioli, Optical gap of CuO, Phys. Rev. B 52, 1433 (1995).

[64] Y. Wang, P. Miska, D. Pilloud, D. Horwat, F. Mücklich, and J. F. Pierson, Transmittance enhancement and optical band gap 
widening of $\mathrm{Cu}_{2} \mathrm{O}$ thin films after air annealing, J. Appl. Phys. 115, 073505 (2014).

[65] H.-s. Kim, M. David, W.-h. Park, M. Patel, and J. Kim, $\mathrm{Cu}_{4} \mathrm{O}_{3}$ based all metal oxides for transparent photodetectors, Sens. Actuators, A 253, 35 (2016).

[66] D. Tahir and S. Tougaard, Electronic and optical properties of $\mathrm{Cu}, \mathrm{CuO}$ and $\mathrm{Cu}_{2} \mathrm{O}$ studied by electron spectroscopy, J. Phys.: Condens. Matter 24, 175002 (2012).

[67] M. A. Green, Self-consistent optical parameters of intrinsic silicon at $300 \mathrm{~K}$ including temperature coefficients, Sol. Energy Mater. Sol. Cells 92, 1305 (2008).

[68] L. C. Olsen, R. C. Bohara, and M. W. Urie, Explanation for lowefficiency $\mathrm{Cu}_{2} \mathrm{O}$ Schottkybarrier solar cells, Appl. Phys. Lett. 34, 47 (1979).

[69] S. Masudy-Panah, G. K. Dalapati, K. Radhakrishnan, A. Kumar, H. R. Tan, E. Naveen Kumar, C. Vijila, C. C. Tan, and D.
Chi, $p \mathrm{CuO} / n \mathrm{Si}$ heterojunction solar cells with high open circuit voltage and photocurrent through interfacial engineering, Prog. Photovoltaics Res. Appl. 23, 637 (2015); S. Dongaonkar and M. A. Alam, Geometrical design of thin film PV modules for improved shade tolerance and performance, arXiv:1303.4604.

[70] F. P. Koffyberg and F. A. Benko, A photoelectrochemical determination of the position of the conduction and valence band edges of ptype $\mathrm{CuO}$, J. Appl. Phys. 53, 1173 (1982).

[71] Q. Bai, W. Wang, Q. Zhang, and M. Tao, n-type doping in $\mathrm{Cu}_{2} \mathrm{O}$ with $\mathrm{F}, \mathrm{Cl}$, and $\mathrm{Br}$ : A first-principles study, J. Appl. Phys. 111, 023709 (2012).

[72] A. Živković , A. Roldan, N. De Leeuw, Research for "Copper oxide for potential photovoltaic application - density functional theory simulation datasets", http://doi.org/10.17035/d. 2017.0043151204 (2018). 